\title{
Multidisciplinary Treatment of Mesiodens Causing Delayed Tooth Eruption and Follow-Up: Report of Two Cases
}

\author{
Nihal Altunok Unlu' Ahmet Altan ${ }^{2}$ Halenur Altan ${ }^{1}$ \\ 'Department of Pediatric Dentistry, Faculty of Dentistry, \\ Gaziosmanpasa University, Tokat, Turkey \\ ${ }^{2}$ Department of Oral and Maxillofacial Surgery, Faculty of Dentistry, \\ Gaziosmanpasa University, Tokat, Turkey
}

\begin{abstract}
Address for correspondence: Nihal Altunok Unlu, DDS, Department of Pediatric Dentistry, Faculty of Dentistry, Gaziosmanpasa University, Tokat 60100, Turkey

E-mail: altunoknihal@gmail.com
\end{abstract}

\begin{abstract}
Mesiodens is a common dental anomaly. It is mostly observed in the upper anterior and lower premolar regions. Mesiodens' existence causes complications such as diastema, delayed eruption, rotation, root resorption, and dentigerous cyst formation. These cases aim to present the treatment and follow-up of mesiodens, which caused delayed tooth eruption.

Mesiodens treatment is a controversial issue. Early diagnosis and multidisciplinary treatment of the complicating mesiodens may prevent further orthodontic treatment.
\end{abstract}

Keywords: Mesiodens, orthodontic extrusions, supernumerary tooth

\section{Introduction}

Supernumerary teeth may be defined as extra teeth, more than a normal number of teeth. This is a dental abnormality, also called hyperdontia, that affects the number of teeth.[1] The supernumerary teeth can be developed in almost any area of dental arches.[2] The frequency with which this problem is seen is reported to be between $0.15 \%$ and $3.9 \% .[3,4]$ While supernumerary teeth can be seen in both primary and permanent dentitions, the chance of having them in the permanent dentition is five times more frequent.[3] A supernumerary tooth located in the maxillary central incisor region is known as a mesiodens, which has an overall prevalence between $0.15 \%$ and $1.9 \%$. Mesiodens can change the eruption path and the permanent incisors' positions to differ considerably in both occlusion and appearance.[5]

Mesiodens can be classified as conical, tuberculate, and supplemental. Conical mesiodens is the most common form that usually occurs as a single mesiodens between the two maxillary central incisors in the palatal area.[6] They typically appear in a single form. Where tuberculate shaped mesiodens is commonly seen is the permanent dentition, and the mesiodens disrupt eruption.[6,7] Root formation does not exist in conicalshaped teeth, while it also does not exist or is incomplete in tuberculate-shaped teeth. Supplemental mesiodens is most commonly seen in the primary dentition.[6] These tend to look like the normal series of teeth and barely encounter eruption.

How to cite this article: Altunok Unlu N, Altan A, Altan H. Multidisciplinary Treatment of Mesiodens Causing Delayed Tooth Eruption and Follow-Up: Report of Two Cases. J Pediatr Dent 2020;6(2):46-52 
Several clinical complications might occur due to mesiodens such as involvement with the adjacent teeth eruption and location, mesiodens pulpitis, dentigerous cyst formation, delayed or non-eruption of maxillary incisors, axial rotation or inclination of erupted permanent teeth, intraoral infection, and midline diastema.[7-10] The most frequently covered complications in the literature and the most commonly reported complications are diastema, delayed eruption, rotation, root resorption, and dentigerous cyst formation.[7-8]

We aimed to give information about the surgical intervention's application time and success and removable orthodontic apparatus in mesiodens treatment.

\section{Case Presentation}

\section{Case 1}

A 9.5-year-old girl was referred to our clinic with the complaint of a smaller anterior tooth. Anamnesis revealed that the patient did not have any disease and did not use any medication. The clinical examination was indicated that the patient was in mixed dentition. Tooth 11,12,22 have erupted, but tooth 61 was overretained. (Fig. 1a). Radiological examination revealed a mesiodens at the level of tooth 21 (Fig. 1d, 1e). CBCT was taken to determine the position of mesiodens and thickness of bone.

As a result of consultation with Oral and Maxillofacial Surgery, it was decided that the mesiodens was in the eruption path, tooth 21 could not be erupted spontaneously, and the mesiodens had to be surgically removed to enable the impacted tooth 21 to erupt. The treatment was planned in two stages as surgical intervention and orthodontic eruption for the tooth's eruption via an apparatus. The verbal and written informed consent form was taken from the patient.

\section{Surgical Intervention}

At the first stage of the treatment, a closed maintenance technique was decided to enable the teeth to erupt. Tooth 61 and mesiodens was extracted under local anesthesia. An incision was made on the vestibular mucosa. After a blunt dissection with the help of a periosteal elevator, mesiodens was removed. The chain button was bonded to the third of the labial surfaces of the crown. Hemostasis was achieved, and the wound was closed with a 3.0 silk suture (SILK, Boz, Turkey). The chain was positioned to the outside of the suture (Fig. $1 b, 1 c)$. The patient's postoperative period was uneventful, and one week after the operation, the patient's sutures were removed.
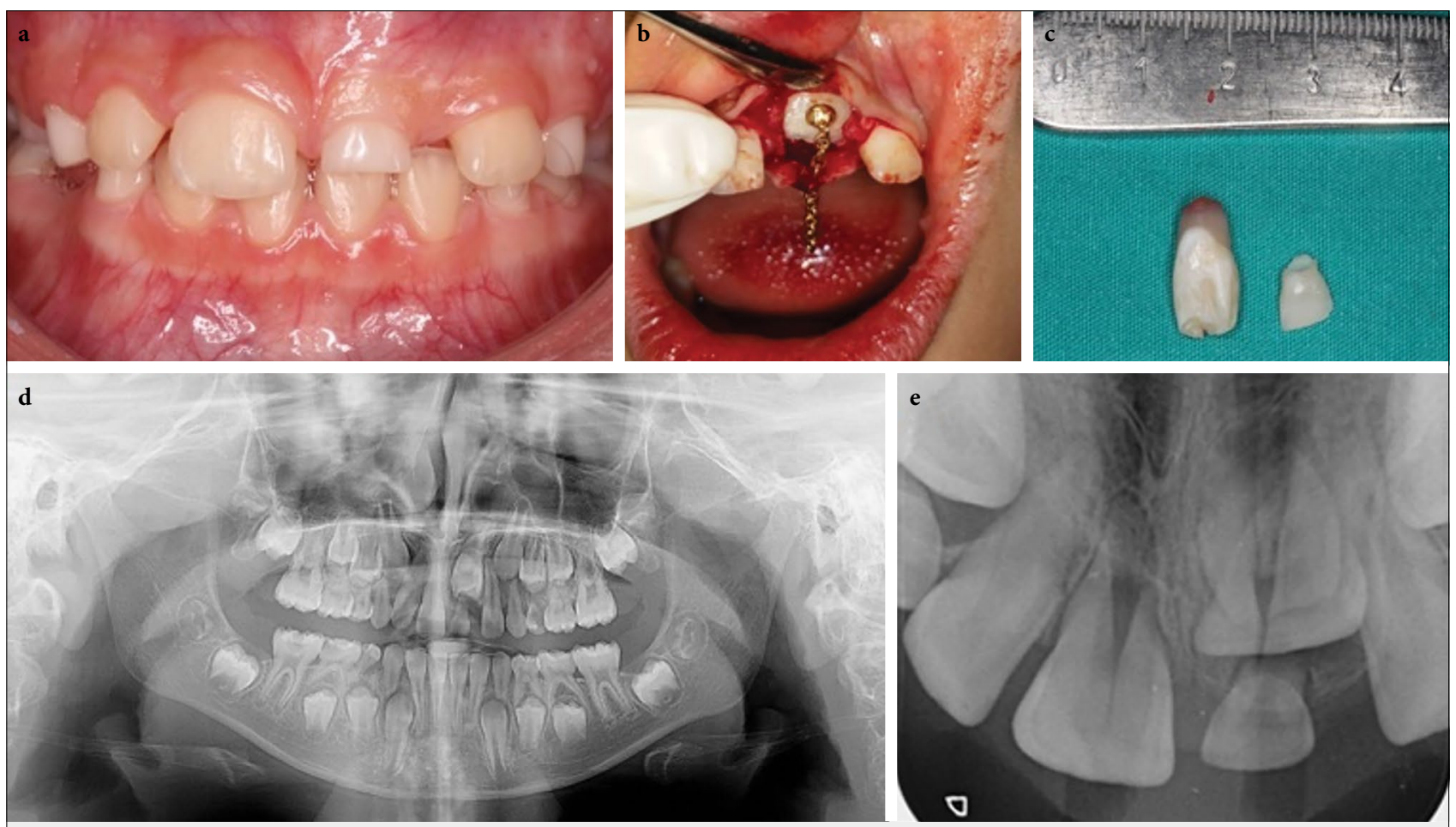

Figure 1. (a) Preoperative intraoral photography. (b, c) Photography of surgical operation and placement of the button to the \#21. (d, e) Preoperative panoramic radiograph of the patient and maxillary periapical radiograph showing mesiodens, causing the permanent central incisor's eruption disturbance 

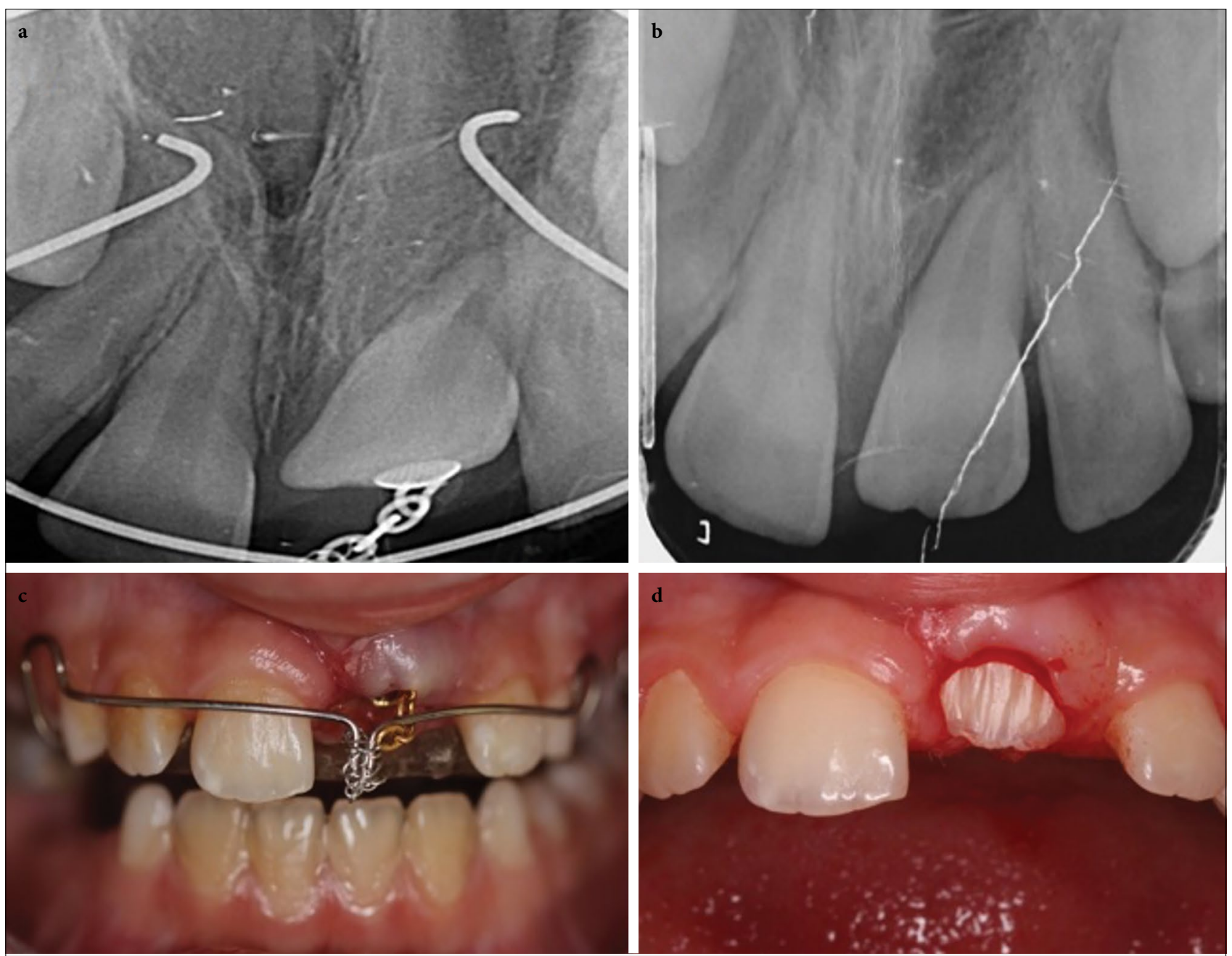

Figure 2. (a, b) Radiography of orthodontic extrusion with chain and after tooth eruption. (c) Photography of orthodontic extrusion with chain. (d) The button on the vestibule surface was removed after the impacted tooth erupted

\section{Orthodontic Intervention}

An apparatus was designed with a spring to which ligature wire could be attached to enable the tooth to erupt. The apparatus was adjusted so that the springs would fit the lower vestibular sulcus without causing any trauma when the patient placed it into her mouth. The chain was stretched with support from the apparatus, and the tooth-eruption process started (Fig. 2c). As the impacted tooth erupted, the ligature wire was cut, and the process progressed until the tooth erupted totally. The eruption process took four months. The movement of the teeth was followed by clinical examination and periapical radiography (Fig. 2a, 2b). The button on the vestibule surface was removed after the impacted tooth erupted (Fig. 2d).

As the tooth erupted in a palatinate position, the cross apparatus was made, and tooth 21 was positioned forward, and then the use of the apparatus was ended (Fig. 3). The patient is still being followed up (Fig. 4).

\section{Case 2}

A 9-year-old girl applied to our clinic with the complaint that her other front tooth did not erupt. Anamnesis revealed that the patient did not have any disease and did not use any medication. The clinical examination was revealed that the patient was in mixed dentition. Tooth 11,12,22 have erupted, but tooth 61 was over-retained because of mesiodens. Radiological examination revealed a mesiodens at the level of tooth 21 (Fig. 5a, 5b). CBCT was taken to determine the position of mesiodens and thickness of bone.

As a result of the consultation with Oral and Maxillofacial Surgery, it was decided to remove mesiodens and tooth 61 , as in case 1 . The treatment was planned in two stages as surgical intervention and the tooth's eruption via an apparatus. The verbal and written informed consent form was taken from the patient. 


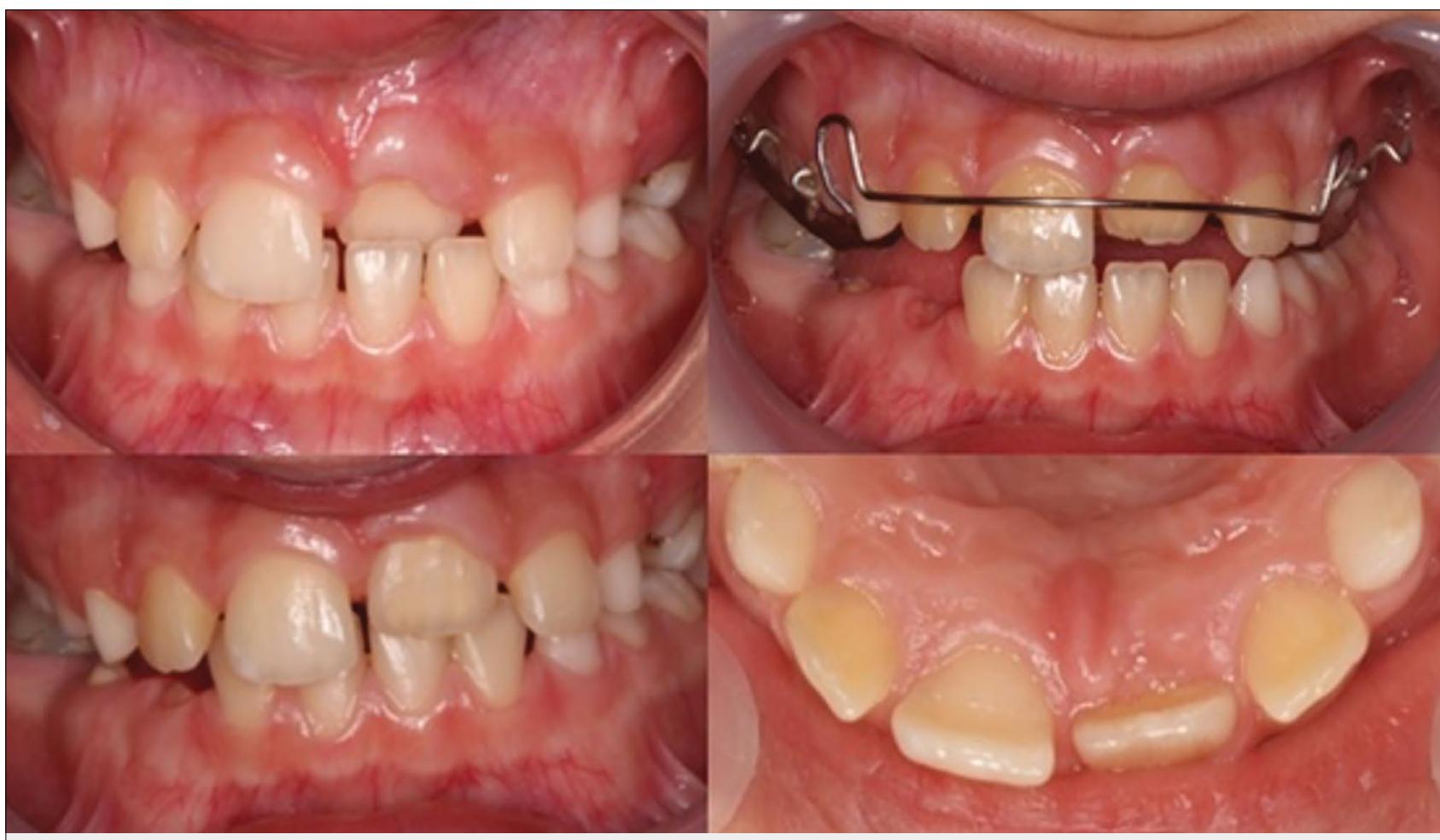

Figure 3. Tooth \#21 was positioned forward with cross-apparatus
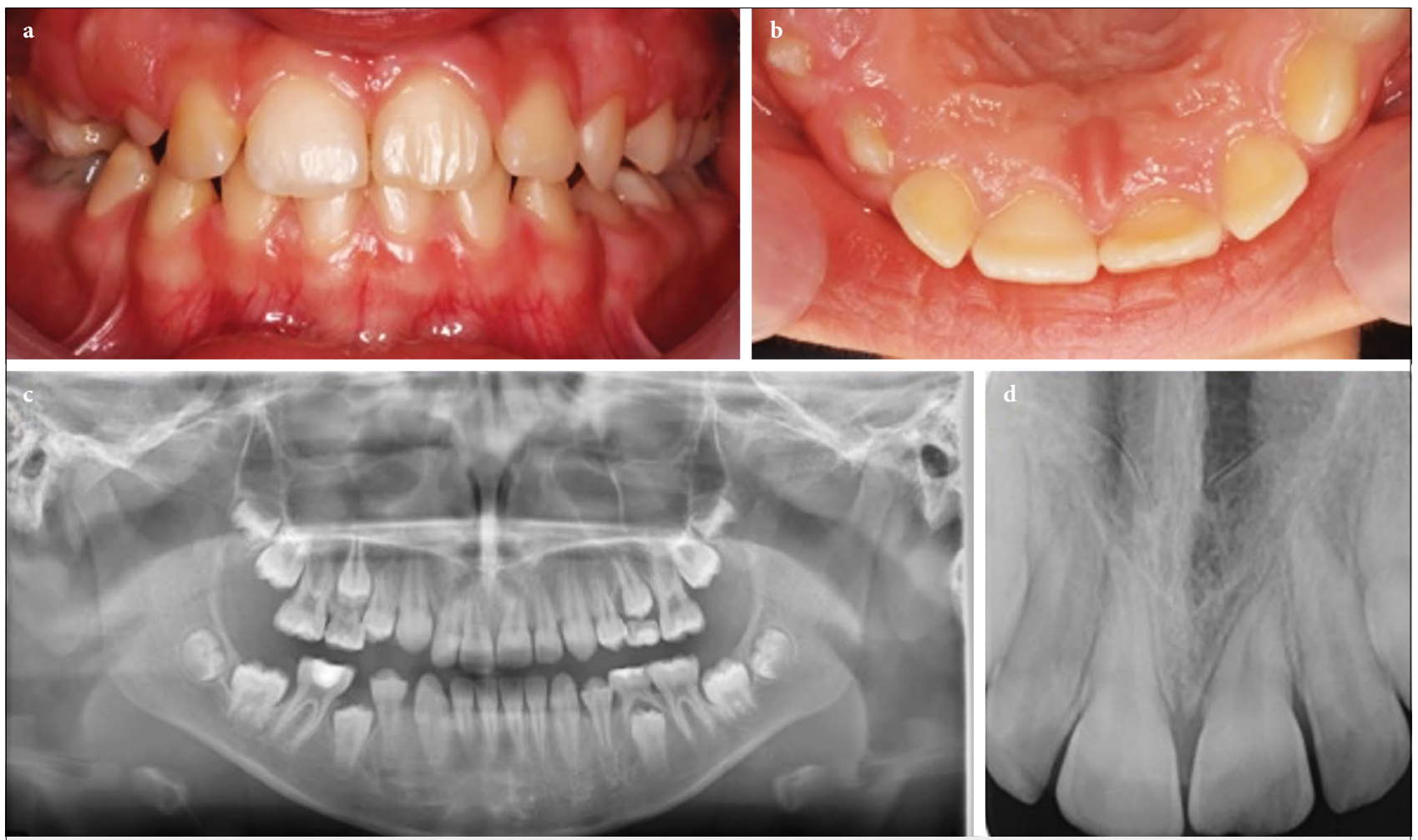

Figure 4. $(a, b)$ Intraoral photography at 15th month. $(c, d)$ Panoramic radiograph of the patient and maxillary periapical radiograph at $15^{\text {th }}$ month

\section{Surgical Intervention}

The surgical intervention was planned and applied, as in case 1 . The chain button was bonded to the third of the labial surfaces of the crown. Hemostasis was achieved, and the wound was closed with a 3.0 silk suture. The chain was positioned to the outside of the 


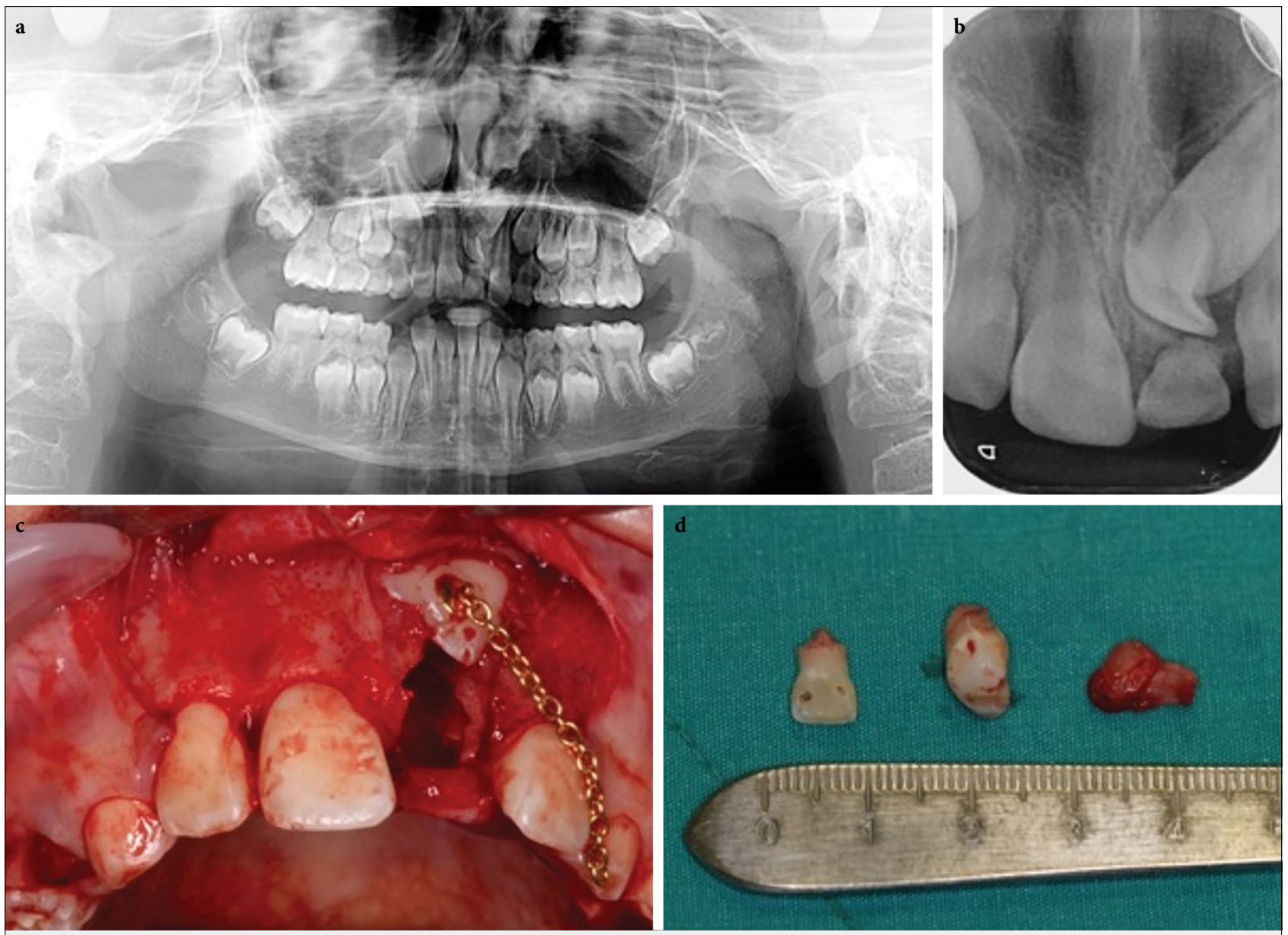

Figure 5. (a, b) Diagnostic radiography. (c, d) After extracting tooth \#61 and the mesiodens, the chain button was attached to the tooth $\# 21$

suture (Fig. 5c, 5d). After one week of the operation, the patient's sutures were removed.

\section{Orthodontic Intervention}

The apparatus was planned and prepared, as in case 1 . The chain was supported by the spring and tightened, and the tooth-eruption process started (Fig. 6a, 6b).

Due to the delay in the eruption of tooth 21 , the design of the apparatus was changed, and support was obtained from the mandibula for better anchorage. Mesiodistal spring was applied to tooth 22 in order to provide space for tooth 21. (Fig. 6c).

The tooth was seen in the mouth 10 months later due to the position of tooth 21. Afterward, her spontaneous eruption was expected, and she was called for controls. (Fig. 7). Due to the lack of space in the arch for tooth 21 , the patient was transferred to the orthodontics department.

\section{Discussion}

Mesiodens treatment is a controversial issue for dentists. Three methods can be used to manage the mesiodens: spontaneous eruption, early intervention, and delayed intervention.[11,12] For a spontaneous eruption, three factors can be considered a priority for the expecting period. Such factors are the type of mesiodens, the degree of the apical closure of the unerupted permanent tooth, and having enough space for the permanent tooth to erupt.[10-12]

In these cases, as the upper central teeth' roots were closed, and the mesiodens was located on the eruption path, the spontaneous eruption was not seen as possible. This period is called "late intervention," and the impacted permanent tooth needs to erupt via surgical removal of mesiodens and orthodontic treatment. Conversely, it has also been reported that the impacted tooth was left to spontaneous eruption after the supernumerary tooth was removed.[13] Due to the probability of eruption failure, we choose the less conservative intervention to expose the impacted tooth at the time of surgery and begin orthodontic traction.

According to the algorithm in this study by Dmytrenko et al[14], the impacted tooth crown should be exposed surgically in the treatment planning of deep 

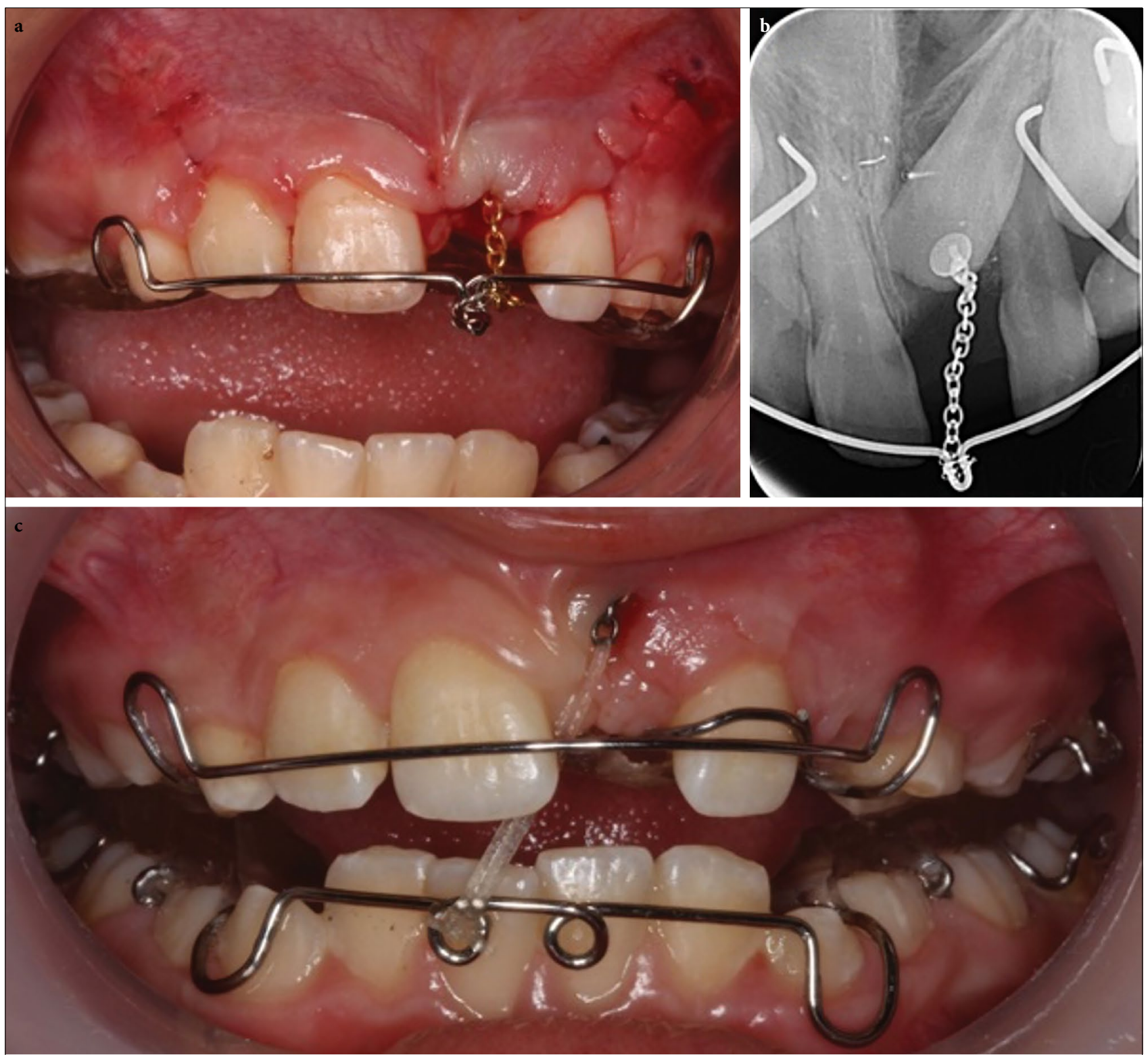

Figure 6. (a, b) Sutures were removed one week after the surgery, and the application of force was started on the tooth \#21 with support from the appliance. (c) To increase the force due to the delay in the eruption of the tooth \#21, the apparatus design was changed, and support was taken from the mandibula. Mesiodistal spring was applied to tooth \#22 in order to provide space for tooth \#21

supernumerary teeth in early mixed dentition. Orthodontic force should be applied to the impacted tooth, and this force can be applied with removable (special spring designs) or fixed orthodontic apparatus (bracket-ligature splint, bracket system, mini implant). [14] The mini implant's major disadvantage is the possibility of damage to the underlying permanent tooth germ or root.[15] The bracket system was not preferred because the patients were in the mixed dentition period.

One of the most important points to be considered regarding the eruption of impacted teeth with an apparatus is the intensity and direction of application of the force to be applied. In the first case, the light forces and the application's direction were adjusted parallel to the tooth's axial line. In the second case, the direction of force was initially parallel to the tooth's axial axis. In the second appliance design, the force's application direction was set the first mesiovertically and then distovertically to bypass the root of the lateral tooth.

\section{Conclusion}

The impacted anterior tooth in a child creates an aesthetic concern, an undeniable problem for early life. The treatment of the impacted tooth gives to the child increases self-confidence and enhances confidence. To 

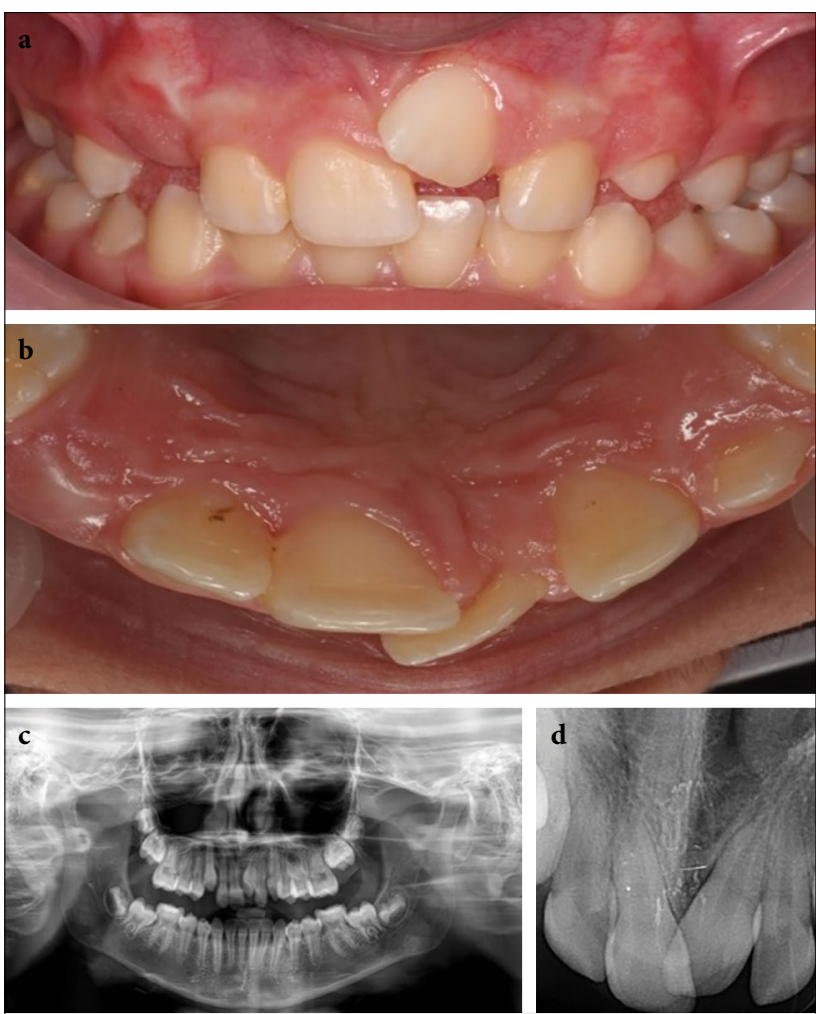

Figure 7. (a, b) Intraoral photographs of the patient at the $15^{\text {th }}$-month control. (c, d) Panoramic and periapical radiographs at the 15th-month follow-up of the patient

conclude, when complications are detected, an early response might lead to fewer surgical and orthodontic interventions subsequently.

\section{Financial Disclosure: Nil.}

Conflict of Interest: None declared.

\section{References}

1. Kasimoglu Y, Koruyucu M, Seymen F. Characteristics of non-syndromic supernumerary teeth in (a group of Turkish) children. Int J Med Invest 2020;9(1):50-59

2. Luten JR Jr. The prevalence of supernumerary teeth in primary and mixed dentitions. J Dent Child 1967;34(5):346-353
3. Brabant H. Comparison of the characteristics and anomalies of the deciduous and the permanent dentition. J Dent Res 1967;46(5):897-902

4. McKibben DR, Brearley LJ. Radiographic determination of the prevalence of selected dental anomalies in children. ASDC J Dent Child 1971;38(6):390-398

5. Kim Y, Jeong T, Kim J, Shin J, Kim S. Effects of mesiodens on adjacent permanent teeth: a retrospective study in Korean children based on cone-beam computed tomography. Int J Paediatr Dent 2018;28(2):161-169 doi:10.1111/ipd.12317

6. Foster TD, Taylor GS. Characteristics of supernumerery teeth in the upper central incisor region. Dent Pract Dent Rec 1969;20(1):8-12

7. Altan H, Akkoc S, Altan A. Radiographic characteristics of mesiodens in a non-syndromic pediatric population in the Black Sea region. J Investig Clin Dent 2019;10(1):e12377 doi:10.1111/ jicd. 12377

8. Primosch RE. Anterior supernumerary teeth--assessment and surgical intervention in children. Pediatr Dent 1981;3(2):204-215

9. Soxman JA. Diagnosis and management of mesiodentes. Gen Dent 2015;63(5):18-19

10. Omer RS, Anthonappa RP, King NM. Determination of the optimum time for surgical removal of unerupted anterior supernumerary teeth. Pediatr Dent 2010;32(1):14-20

11. Russell KA, Folwarczna MA. Mesiodens--diagnosis and management of a common supernumerary tooth. J Can Dent Assoc 2003;69(6):362-366

12. Rotberg SJ, Kopel HM. Early versus late removal of mesiodens: a clinical study of 375 children. Compend Contin Educ Dent 1984;2:115-119

13. Tomizawa M, Otsuka Y, Noda T. Clinical observations of odontomas in Japanese children: 39 cases including one recurrent case. Int J Paediatr Dent 2005;15(1):37-43 doi:10.1111/j.1365263X.2005.00607.x

14. Dmytrenko MI, Gurzhiy OV. Treatment algorithms of patients with impaction of maxillary central incisors caused by supernumerary teeth. Wiad Lek 2018;71(4):922-932

15. Kamatham R, Avisa P, Vinnakota DN, Nuvvula S. Adverse effects of implants in children and adolescents: A systematic review. J Clin Pediatr Dent 2019;43(2):69-77 doi:10.17796/1053-462543.2.1 